\title{
Antiferromagnetism in four dimensions: search for non-triviality*
}

\author{
J. L. Alonso ${ }^{\text {, }}$, H. G. Ballesteros ${ }^{\mathrm{b}}$, I. Campos ${ }^{\mathrm{a}}$, J. M. Carmona ${ }^{\mathrm{a}}$, J. Clemente Gallardo ${ }^{\mathrm{a}}$, \\ L. A. Fernández ${ }^{b}$, D. Iñiguez ${ }^{a}$, V. Martín-Mayor ${ }^{b}$, A. Muñoz Sudupe ${ }^{b}$, A. Tarancón ${ }^{a}$ and C. L. Ullod ${ }^{a}$ \\ ${ }^{a}$ Departamento de Física Teórica, Facultad de Ciencias, \\ Universidad de Zaragoza, 50009 Zaragoza, Spain

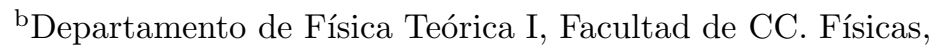 \\ Universidad Complutense de Madrid, 28040 Madrid, Spain
}

\begin{abstract}
We present antiferromagnetism as a mechanism capable of modifying substantially the phase diagram and the critical behaviour of statistical mechanical models. This is particularly relevant in four dimensions, due to the connection between second order transition points and the continuum limit as a quantum field theory.

We study three models with an antiferromagnetic interaction: the Ising and the $\mathrm{O}(4)$ Models with a second neighbour negative coupling, and the $\mathbf{R P}^{2}$ Model. Different conclusions are obtained depending on the model.
\end{abstract}

An antiferromagnetic (AFM) system shows in general different critical behaviour from that of its ferromagnetic counterpart, as will be seen below. This can be used to study the triviality problem in Quantum Field Theory, by computing the critical exponents or the renormalized coupling, which is expressed in terms of the Binder cumulant $U_{L}$ of the model as

$g_{R}=\lim _{L \rightarrow \infty} g_{L}^{(4)}=\lim _{L \rightarrow \infty}\left(L / \xi_{L}\right)^{d} U_{L}$,

and should be different from zero in a non-trivial theory.

One way by which one could hope to see new critical behaviour, i.e. new universality classes, is by considering AFM models. Actually, it has been shown in low dimensions that antiferromagnetism can produce new critical behaviour. For example, in the Ising Model we can introduce it by means of a second-neighbour (related with higher derivatives in the action) negative coupling, which produces frustration in the system. This makes a new ground state to appear, which in $2 d$ gives a new transition line with new critical behaviour [1].

The $3 d \mathbf{R P}^{2}$ AFM Model has also recently been

\footnotetext{
*Presented by J. M. Carmona. Partially supported by CICyT (Spain) AEN93-0604-C03, AEN94-0218, and AEN95-1284E.
}

studied [2]. The Hamiltonian is given by

$\mathcal{H}=-\beta \sum_{<i j>}\left(\boldsymbol{v}_{i} \cdot \boldsymbol{v}_{j}\right)^{2}$,

where $v_{i}$ is a three component normalized vector. Here a negative coupling produces a mixed ground state, formed by one sub-lattice oriented in a fixed direction and the other lying on the plane perpendicular to it, which brings about a new universality class.

We have considered the following models in $4 d$.

\section{AFM Ising Model}

This is the simplest AFM model: the Ising Model in a hypercubic lattice with first- and second-neighbour couplings

$\mathcal{H}=-\beta_{1} \sum_{\boldsymbol{n}, \mu} \sigma_{\boldsymbol{n}} \sigma_{\boldsymbol{n}+\hat{\boldsymbol{\mu}}}-\beta_{2} \sum_{\boldsymbol{n}, \mu<\nu} \sigma_{\boldsymbol{n}} \sigma_{\boldsymbol{n}+\hat{\boldsymbol{\mu}}+\hat{\boldsymbol{\nu}}}$.

It was simulated on a $V=L^{4}$ lattice with periodic boundary conditions, using a Heat Bath update (clusters algorithms are not effective in most of AFM models). The Finite Size Scaling (FSS) was studied up to $L=24$ [3].

This model turns out to have a rich phase diagram, with different vacuum structures. There is a region of ferromagnetic order $(\mathrm{FM}): \sigma_{\boldsymbol{n}}=\sigma_{\mathbf{0}}$ ( $\sigma_{0}$ stands for a fixed spin); there is also a 


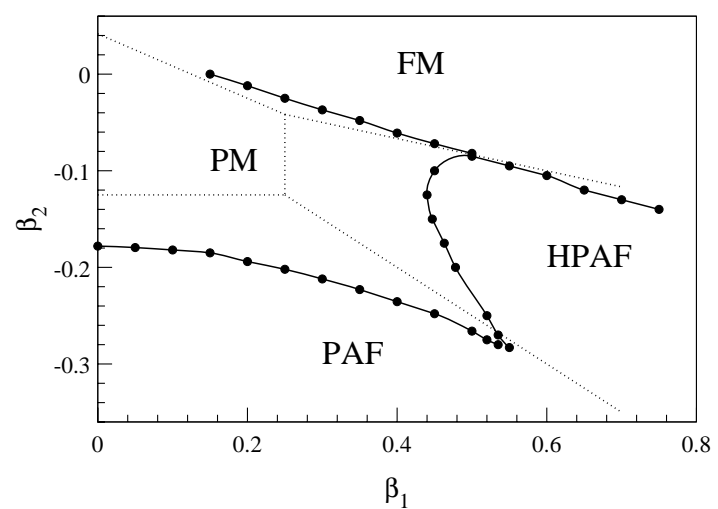

Figure 1. Phase Diagram of the $4 d$ AFM Ising Model.

phase, HPAF, which consists of a FM configuration on a three-dimensional cube and AFM on the other direction $\mu$ : $\sigma_{\boldsymbol{n}}=(-1)^{n_{\mu}} \sigma_{\mathbf{0}}$; finally, in the PAF phase, we have FM on a twodimensional plane and AFM on the other two directions: $\sigma_{\boldsymbol{n}}=(-1)^{n_{\mu}+n_{\nu}} \sigma_{\mathbf{0}}$, where $\mu, \nu(\mu<\nu)$ can be any directions. We define order parameters according to these structures.

The phase diagram is shown in Fig. 1: PM means a disordered phase and the dotted lines are the transition lines given by Mean Field theory. The transition line connected with the usual Ising point $\left(\beta_{2}=0\right)$ is second order with the same (classical) exponents. The PM-HPAF line is first order. And we find another line, PM-PAF, which is disconnected from the Ising critical point. This transition is clearly first order for values of $\beta_{1}$ greater than 0.2 , but the behaviour is not so clear for $\beta_{1}<0.2$.

We produced a great amount of data at the points $\beta_{1}=0.1,0.05,0$. At $\beta_{1}=0$ the system decouples into two independent sublattices, known as $F_{4}$ lattices in the literature. We found that the two peaks characteristic of a first order transition are only distinguished at $\beta_{1}=0$, and with the maximum size, $L=24$.

This AFM model teaches us that we have to be very careful, because first order transitions with a very large correlation length can deceive you if you do not go to sufficiently large lattice sizes to see the real behaviour. With smaller sizes, appar- ent second order transitions arise, and even false critical exponents can be measured. This system behaves for small $L$ as a weak first order transition, and presents the pseudo critical exponent $\alpha / \nu=1$, precursor of a first order behaviour for large lattice sizes [4].

\section{AFM RP ${ }^{2}$ Model}

This is the four-dimensional version of the model defined in (2). It was simulated by combining Metropolis and Over-relaxed algorithms, up to $L=24$ lattice sizes 6 .

Two order parameters appear due to the special nature of the ground state, one FM and the other AFM (or staggered). They are defined through tensors attached to every lattice site

$\mathrm{T}_{i}^{\alpha \beta}=v_{i}^{\alpha} v_{i}^{\beta}-\frac{1}{3} \delta^{\alpha \beta}$

as the normal or staggered sum of these tensors to the whole volume.

In order to calculate critical exponents, we use the FSS ansatz that allows to write

$Q_{O} \equiv \frac{\langle O(2 L, \beta)\rangle}{\langle O(L, \beta)\rangle}=2^{x_{O} / \nu} \frac{F_{O}(\xi(2 L, \beta) / 2 L)}{F_{O}(\xi(L, \beta) / L)}$

(except for scaling corrections), so that

$$
\left.Q_{O}\right|_{Q_{\xi}=2}=2^{x_{O} / \nu}+\cdots,
$$

from where the critical exponent $x_{O}$ is readily extracted.

The model has a second order transition at $\beta \approx-1.34$, its critical exponents being almost gaussian, the differences likely due to logarithmic corrections. To know about the triviality of

Table 1

$g_{R}\left(L, \beta_{c}(\infty)\right)$ for the $4 d$ AFM $\mathbf{R P}^{2}$ Model.

\begin{tabular}{rl}
\hline$L$ & $g_{R}$ \\
\hline \hline 8 & $3.16(3)$ \\
10 & $2.84(4)$ \\
12 & $2.61(5)$ \\
16 & $2.34(4)$ \\
20 & $2.08(9)$ \\
24 & $1.95(13)$ \\
\hline
\end{tabular}


the theory we should therefore look at the renormalized coupling, defined by (11), at the critical temperature. As can be seen in Table 1, for the staggered sector the $g_{R}$ gets smaller values as the lattice size grows. We obtain a good fit to $1 / \log L$ without a constant term, so that the theory seems to be trivial. However, in the ferromagnetic sector, $g_{R}$ takes a negative, rather stable, value. This might be an indication of a non-trivial limit for this theory.

\section{AFM O(4) Model}

We consider a system of spins $\left\{\boldsymbol{\Phi}_{\boldsymbol{n}}\right\}$ taking values in the hyper-sphere $S^{3} \subset \mathbf{R}^{4}$ and placed in the nodes of a hypercubic lattice. The interaction is defined by the Hamiltonian:

$$
\mathcal{H}=-\beta_{1} \sum_{\boldsymbol{n}, \mu} \boldsymbol{\Phi}_{\boldsymbol{n}} \boldsymbol{\Phi}_{\boldsymbol{n}+\hat{\boldsymbol{\mu}}}-\beta_{2} \sum_{\boldsymbol{n}, \mu<\nu} \boldsymbol{\Phi}_{\boldsymbol{n}} \boldsymbol{\Phi}_{\boldsymbol{n}+\hat{\boldsymbol{\mu}}+\hat{\boldsymbol{\nu}}}
$$

The phase structure is found to be qualitatively very similar to that of the Ising Model of Fig. 1, but now we have a continuum symmetry. We did Monte Carlo simulations in lattices ranging from $L=6$ to $L=24$ [5]. The update method was a combination of Over-relaxed and Heat-Bath algorithms, being $z \approx 1$.

In the $\mathrm{O}(4)$ AFM Model, the whole phase transition line PM-PAF seems to be second order. We computed the critical exponents on this line at $\beta_{1}=0$ coupling $\left(F_{4}\right.$ lattice) with FSS techniques. The results are quoted in Table 2. The correlation length exponent, $\nu$, is very close to the Mean Field prediction, namely $\nu=0.5$. Although we have not obtained a reliable measure of the specific heat exponent our results point to $\alpha / \nu<1$ and disagree with the expected behaviour for a weak first order transition.

Table 2

Critical Exponents of the $4 d$ AFM O(4) Model.

\begin{tabular}{cccc}
\hline Lattice sizes & $\gamma / \nu$ & $\beta / \nu$ & $\nu$ \\
\hline \hline $6 / 12$ & $2.417(3)$ & $0.791(4)$ & $0.474(10)$ \\
$8 / 16$ & $2.403(3)$ & $0.792(6)$ & $0.483(8)$ \\
$10 / 20$ & $2.410(2)$ & $0.790(4)$ & $0.471(6)$ \\
$12 / 24$ & $2.403(5)$ & $0.797(5)$ & $0.483(7)$ \\
\hline
\end{tabular}

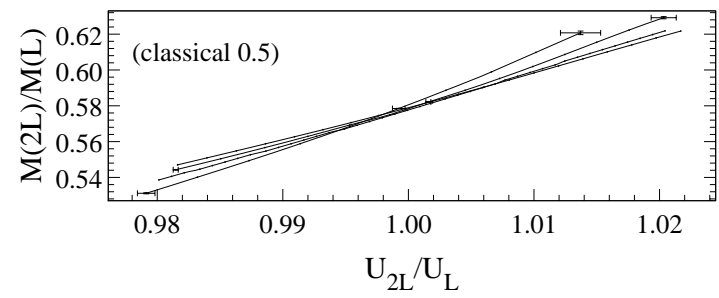

Figure 2. Quotients to obtain $\gamma / \nu(\mathrm{O}(4))$.

From our $\gamma / \nu$ estimation (see Fig. 2) (or $\beta / \nu$ using the hyper-scaling relation) the exponent $\eta$ associated with the anomalous dimension of the field is $\eta \approx-0.4$. This fact itself would imply the non-triviality of the theory, but bearing in mind the results of section 1 , transitory behaviour cannot be discarded. However, the stability of our measures of $\gamma / \nu$ for the different lattice sizes, (more than a hundred of standard deviations from $\eta=0$ ), makes this rather unlikely. We also measured the Binder cumulant at the critical point, finding that it stays almost constant when increasing the lattice size, which points to a non-zero value of the renormalized constant in the thermodynamical limit.

\section{REFERENCES}

1. J.L. Morán-López, F. Aguilera-Granja, and J.M. Sánchez, J. Phys.: Cond.Matt. 6 (1994) 9759.

2. H.G. Ballesteros, L.A. Fernández, V. MartínMayor, and A. Muñoz Sudupe, Phys. Lett. B378 (1996) 207; hep-lat/9605037, and these proceedings.

3. J.L. Alonso, J.M. Carmona, J. Clemente Gallardo, L.A. Fernández, D. Iñiguez, A. Tarancón and C.L. Ullod, Phys. Lett. B376 (1996) 148.

4. L.A. Fernández, M.P. Lombardo, J.J. RuizLorenzo, and A. Tarancón, Phys. Lett. B227 (1992) 485.

5. I. Campos, L.A. Fernández, and A. Tarancón, preprint hep-lat/9606017.

6. H. G. Ballesteros, J. M. Carmona, L. A. Fernández, V. Martín-Mayor,A. Muñoz Sudupe and A. Tarancón, in preparation. 